Copyright (C 2014 IEEE. Personal use of this material is permitted. Permission from IEEE must be obtained for all other uses, in any current or future media, including reprinting/republishing this material for advertising or promotional purposes, creating new collective works, for resale or redistribution to servers or lists, or reuse of any copyrighted component of this work in other works. 


\title{
New results in impulse-free continuous-time cheap LQ optimal control
}

\author{
Augusto Ferrante and Lorenzo Ntogramatzidis
}

\begin{abstract}
In this paper, we provide conditions for the solvability of LQ (linear-quadratic) optimal control problems in which the input function is not penalised in the performance index. In particular, we focus our attention on regular input functions, i.e., on the control functions that do not involve distributions, which in general appear in the solution of singular problems.
\end{abstract}

\section{INTRODUCTION}

It is well known that the solvability of the classic finite and infinite-horizon LQ optimal control problem strongly depends on the matrix weighting the input in the cost function, traditionally denoted by $R$. When $R$ is positive definite, the problem is said to be regular (see e.g. [1], [14]), whereas when $R$ is positive semidefinite, the problem is called singular. In the particular case in which $R$ is the zero matrix, the corresponding LQ problem is called cheap. This subject has been dealt with by using the theory of the geometric approach (see for example [12], [24], [18] and references therein). In particular, in [12] and [24] it has been shown that an optimal solution to the singular problem always exists if the class of allowable controls is extended to include distributions. A geometric algorithm is used to isolate the regular (i.e., impulse-free) part of the optimal control, which can be calculated resorting to a reduced order algebraic Riccati equation. The approach presented in [18] is based on an analysis of the structure of both singular and cheap problems (where the latter is treated as the limiting case of the former), by the exploitation of the so-called special coordinate basis. Valuable results on this subject have also been presented in [19], [21], [20], which are based on linear matrix inequalities.

A different approach for the cheap LQ problem was introduced in [16], where geometric techniques were used to recast the cheap control problem as a perfect decoupling problem, to the end of characterising in strict geometric terms the subspace of initial conditions for which the optimal control is non-impulsive.

The recent papers [9], [10], have investigated the connection of the generalised continuous-time algebraic Riccati

Partially supported by the University of Padova under grant $n$. CPDA111238 "A Unifying Framework for Spectral Estimation and Matrix Completion: A New Paradigm for Identification, Estimation, and Signal Processing" and by the Australian Research Council under the grant FT120100604.

A. Ferrante is with Dipartimento di Ingegneria dell'Informazione, Università di Padova, via Gradenigo, 6/B - 35131 Padova, Italy, augusto@dei.unipd.it

L. Ntogramatzidis is with the Department of Mathematics and Statistics, Curtin University, Perth (WA), Australia. L.Ntogramatzidis@curtin.edu.au equation - which is defined by substitution of the inverse of $R$ with the pseudo-inverse in the standard algebraic Riccati equation - and the solution of the singular LQ optimal control problem. In these papers it is shown in particular that whenever the generalised Riccati equation admits a symmetric solution, the corresponding singular LQ problem admits an impulse-free optimal control from any initial condition of the system. Moreover, such control can always be expressed as a state-feedback, where the gain can be obtained from the solution of the generalised Riccati equation. A very interesting question, which remained open in these contributions, is the following: when the singular LQ problem admits a regular solution for all initial states, is the generalised Riccati equation guaranteed to admit at least one symmetric positive semidefinite solution? In this paper, we provide an affirmative answer to this question for the cheap case. Indeed, the first aim of this paper is to show that a cheap LQ problem has an impulse-free optimal control from all initial condition if and only if the corresponding generalised Riccati equation (which in the cheap case reduces to a Lyapunov equation) has a symmetric positive semidefinite solution.

The second main result of this paper is another geometric result related to the solvability of a cheap LQ problem in terms of impulse-free optimal controls.

Notation. The image and the kernel of matrix $M$ are denoted by $\operatorname{im} M$ and $\operatorname{ker} M$, respectively, while the transpose and the Moore-Penrose pseudo-inverse of $M$ are denoted by $M^{\mathrm{T}}$ and $M^{\dagger}$, respectively.

\section{THE CHEAP LQ PROBLEM}

Let $n, m \in \mathbb{N}$ with $m \leq n$. Consider the standard linear time-invariant state differential equation

$$
\dot{x}(t)=A x(t)+B u(t), \quad x(0)=x_{0} \in \mathbb{R}^{n} .
$$

where, for all $t \geq 0, x(t) \in \mathbb{R}^{n}$ represents the state and $u(t) \in$ $\mathbb{R}^{m}$ represents the input function, and where $A \in \mathbb{R}^{n \times n}$ and $B \in \mathbb{R}^{n \times m}$.

The cheap linear quadratic (LQ) problem can be formulated as follows.

Problem 2.1: Find an input function $u(t)$ such that the performance index

$$
J\left(x_{0}, u\right)=\int_{0}^{\infty} x^{\mathrm{T}}(t) Q x(t) d t,
$$

where $Q=Q^{\mathrm{T}} \geq 0$, is minimised, subject to the constraint given by (1). 
Consider for example the scalar dynamical system $\dot{x}(t)=$ $u(t)$ with initial condition $x(0)=x_{0} \neq 0$, along with the performance index (2) with $Q$ equal to the identity matrix. By using a control input $u(t)=-\alpha x(t)$, the value of the performance index can be made arbitrarily close to zero by choosing a sufficiently large value of $\alpha$. But it is clear that no regular control $u$ can annihilate the cost. This example shows that in singular and cheap cases a solution may not exist, since it is impossible to attain the infimum of the performance index. Stated differently, in these cases the value of the performance index can be infimised but not minimised. Nevertheless, for the singular and cheap LQ problem, a solution is guaranteed to exist for all initial conditions only if the class of allowable controls is extended to include distributions. A fairly complete distributional framework was developed in [12] and [24]. In particular, in these papers it was established that in general Problem 2.1 is solvable for any initial condition $x_{0}$ if the control involves a regular part (which can always be written as a static state feedback as in the regular LQ problem), plus a linear combination of Dirac deltas with its derivatives in the sense of distributions.

In this paper we are interested in the characterisation of the cases in which the cheap LQ problem is solvable from any initial condition with a control function that does not involve impulses. In other words, we characterise the situations in which the optimal control can be sought within the class of static state feedback inputs, so that optimal solutions containing distributions are ruled out.

In this paper, we make the following standing assumption.

Assumption 2.1: For every initial condition $x_{0}$ there exists an input function $u(t) \in \mathbb{R}^{m}$, with $t \geq 0$, such that $J\left(x_{0}, u\right)$ in (2) is finite.

The following theorem provides a necessary and sufficient condition for the solvability of Problem 2.1 without the need of distributions.

Theorem 2.1: The cheap LQ problem admits nonimpulsive solutions for all $x_{0} \in \mathbb{R}^{n}$ if and only if the Lyapunov equation

$$
X A+A^{\mathrm{T}} X+Q=0
$$

admits a symmetric positive semi-definite solutions $X$ such that $X B=0$.

Proof: Consider a singular LQ optimal control problem, which consists in the minimisation of the performance index

$$
J^{\prime}\left(x_{0}, u\right)=\int_{0}^{\infty}\left[\begin{array}{ll}
x^{\mathrm{T}}(t) & u^{\mathrm{T}}(t)
\end{array}\right]\left[\begin{array}{cc}
Q & S \\
S^{\mathrm{T}} & R
\end{array}\right]\left[\begin{array}{l}
x(t) \\
u(t)
\end{array}\right] d t
$$

subject to (1), where $Q \in \mathbb{R}^{n \times n}, S \in \mathbb{R}^{n \times m}$ and $R \in \mathbb{R}^{m \times m}$ are such that

$$
\Pi \triangleq\left[\begin{array}{cc}
Q & S \\
S^{\mathrm{T}} & R
\end{array}\right]=\Pi^{\mathrm{T}} \geq 0 .
$$

It was shown in [9, Theorem 4.1] and in [10, Theorem 3.2] that if the generalised algebraic Riccati equation

$$
X A+A^{\mathrm{T}} X-(S+X B) R^{\dagger}\left(S^{\mathrm{T}}+B^{\mathrm{T}} X\right)+Q=0,
$$

subject to the additional constraint

$$
\operatorname{ker} R \subseteq \operatorname{ker}(S+X B),
$$

admits a symmetric positive semidefinite solution, this singular LQ problem admits a regular optimal control for any initial condition. The cheap LQ problem introduced in this paper is a particular case of this problem in which $R=0$. Indeed, if $R=0$, condition (5) implies $S=0$. In this case, taking into account that if $R=0$, the Moore-Penrose pseudoinverse $R^{\dagger}$ is the zero matrix, (6) reduces to (3), and the condition (7) reduces to $X B=0$.

It remains to show that the converse is also true, i.e., if the cheap LQ problem admits non-impulsive solutions for any initial condition, then (3) admits a symmetric positive semi-definite solutions $X$ such that $X B=0$. Consider a factorisation $Q=C^{\mathrm{T}} C$, so that the performance index can be re-written as

$$
J\left(x_{0}, u\right)=\int_{0}^{\infty} y^{\mathrm{T}}(t) y(t) d t
$$

where $y(t)=C x(t)$ can be thought of as a system output. Since it is assumed that the LQ problem admits nonimpulsive solutions for any initial condition, [24] guarantees that for any $x_{0}$ the optimal control function can be written as a static state feedback

$$
u^{*}(t)=-K x(t)
$$

and the closed-loop system is

$$
\left\{\begin{array}{l}
\dot{x}(t)=(A-B K) x(t) \\
y(t)=C x(t)
\end{array}\right.
$$

Let $A_{K} \triangleq A-B K$ denote the closed-loop matrix. The optimal state is $x(t)=e^{A_{K} t} x_{0}$, and therefore the optimal cost is

$$
J^{*}=x_{0}^{\mathrm{T}}\left[\int_{0}^{\infty} e^{A_{K}^{\mathrm{T}} t} Q e^{A_{K} t} d t\right] x_{0} .
$$

Now, consider $x_{0} \in \operatorname{im} B$. Thus, $x_{0}=B z$ for some $z$. Since in this case with the impulsive control $u(t)=-\delta(t) z$ we can obtain $x\left(0^{+}\right)=0$, and consequently the cost becomes zero, even with $u^{*}$ the value of the cost must be zero. Thus

$$
x_{0} \in \operatorname{ker} \int_{0}^{\infty} e^{A_{K}^{\mathrm{T}} t} Q e^{A_{K} t} d t
$$

for all $x_{0} \in \operatorname{im} B$, which also implies

$$
\operatorname{im} B \subseteq \operatorname{ker} \int_{0}^{\infty} e^{A_{K}^{\mathrm{T}} t} Q e^{A_{K} t} d t .
$$

Thus, $C e^{A_{K} t} B=0$ for all $t \geq 0$. This means that the transfer function $C\left(s I_{n}-A_{K}\right)^{-1} B$ is zero, and therefore also the transfer function matrix $C\left(s I_{n}-A\right)^{-1} B$ is equal to zero.

This proves that

$$
\int_{0}^{\infty} e^{A_{K}^{\mathrm{T}} t} Q e^{A_{K} t} d t=\int_{0}^{\infty} e^{A^{\mathrm{T}} t} Q e^{A t} d t .
$$


We define $X \triangleq \int_{0}^{\infty} e^{A^{\mathrm{T}} t} Q e^{A t} d t$. We show that $X$ satisfies (3). Indeed,

$$
\begin{aligned}
X A+A^{\mathrm{T}} X+Q= & \int_{0}^{\infty} e^{A^{\mathrm{T}} t} Q e^{A t} A d t \\
& +\int_{0}^{\infty} A^{\mathrm{T}} e^{A^{\mathrm{T}} t} Q e^{A t} d t+Q
\end{aligned}
$$

i.e.,

$$
X A+A^{\mathrm{T}} X+Q=\int_{0}^{\infty} \frac{d}{d t}\left(e^{A^{\mathrm{T}} t} Q e^{A t}\right) d t+Q .
$$

Since the cost is finite, $\int_{0}^{\infty} e^{A^{\mathrm{T}} t} Q e^{A t} d t$ is finite, so that $e^{A^{\mathrm{T}} t} Q e^{A t} \rightarrow 0$ as $t \rightarrow \infty$. Thus, the latter becomes

$$
X A+A^{\mathrm{T}} X+Q=\left[e^{A^{\mathrm{T}} t} Q e^{A t}\right]_{0}^{\infty}+Q=0,
$$

which proves the claim.

As a direct consequence of the proof of the previous result, we have the following.

Corollary 2.1: If a cheap LQ problem admits an impulsefree solution for any initial condition $x_{0} \in \mathbb{R}^{n}$, the zero input is always optimal.

\section{A GEOMETRIC CHARACTERISATION}

In this section we provide a simple characterisation for the solvability of the cheap LQ problem by means of an impulse-free control.

We first recall some definitions that will be used in this second part of the paper. The reachable subspace is denoted by $\mathscr{R}(A, B)$. It equals the image of the matrix $\left[\begin{array}{lllll}B & A & B & \ldots & A^{n-1} B\end{array}\right]$, and it also coincides with the smallest $A$-invariant subspace containing the subspace spanned by the columns of $B$. Dually, the unobservable subspace is denoted by $\mathscr{N}(A, C)$. It equals the null-space of the matrix $\left[\begin{array}{c}C \\ C A \\ \vdots \\ C A^{n-1}\end{array}\right]$, and it also coincides with the largest $A$-invariant subspace contained in the null-space of $C$.

The geometric setting developed here requires the following definitions: $\mathscr{V}^{\star}$ stands for the largest $(A, B)$-controlled invariant subspace contained in the null-space of $C$. Recall that $\mathscr{V}^{\star}$ is the subspace of all initial states $x_{0} \in \mathbb{R}^{n}$ of (1) for which an input function exists such that the corresponding output is identically zero. For this reason, $\mathscr{V}^{\star}$ is also referred to as the largest output-nulling subspace of the system described by the triple $(A, B, C)$ (where an output-nulling subspace of $(A, B, C)$ is defined as an $(A, B)$-controlled invariant subspace contained in $\operatorname{ker} C)$. The symbol $\mathscr{S}^{\star}$ stands for the smallest $(A, C)$-conditioned invariant subspace containing the image of $B$, and it is also referred to as the smallest input-containing subspace of the triple $(A, B, C)$ (where an input-containing subspace is an $(A, C)$-conditioned invariant subspace containing $\operatorname{im} B$ ). For a detailed discussion on controlled and conditioned invariant subspaces we refer to the textbooks [2], [22], [25].

Controlled and conditioned invariance are dual concepts, in the sense that, given a triple $(A, B, C)$, a subspace is an $(A, B)$-controlled invariant subspace contained in $\operatorname{ker} C$ if and only if its orthogonal complement is an $(A, C)$-conditioned invariant subspace containing $\operatorname{im} B$, see e.g. [2, p.209]. Recall that, given a friend $F$ of $\mathscr{V}^{\star}$, i.e. a matrix such that $(A+B F) \mathscr{V}^{\star} \subseteq \mathscr{V}^{\star}$, the eigenvalues of $A+B F$ restricted to $\mathscr{V}^{\star}$ are split into two sets. The eigenvalues of $A+B F$ which are restricted to $\mathscr{V}^{\star} \cap \mathscr{S}^{\star}$ are all freely assignable by a suitable choice of $F$. The eigenvalues of the mapping induced by $A+B F$ on the quotient $\mathscr{V}^{\star} / \mathscr{V}^{\star} \cap \mathscr{S}^{\star}$ are fixed for any friend of $\mathscr{V}^{\star}$. The subspace $\mathscr{R}^{\star} \triangleq \mathscr{V}^{\star} \cap \mathscr{S}^{\star}$ can be interpreted as the subspace of states that can be reached from the origin with state trajectories all contained in $\mathscr{V}^{\star}$ (hence invisible at the output), so that it is often referred to as the reachability subspace on $\mathscr{V}^{\star}$. For a detailed analysis of the internal and external eigenstructure of a controlled invariant subspace we refer to [2, pp.217-222] and [22, pp.89-96].

It is easy to see that there holds

$$
\mathscr{V}^{\star}=\max _{F \in \mathbb{R}^{n \times m}} \mathscr{N}(A+B F, C) .
$$

Indeed, $\mathscr{N}(A+B F, C)$ is the largest $(A+B F)$-invariant subspace contained in $\operatorname{ker} C$ for all $F \in \mathbb{R}^{n \times m}$. This means that $\mathscr{N}(A+B F, C)$ is an output-nulling subspace of $\Sigma$ for all $F \in \mathbb{R}^{n \times m}$. However, $\mathscr{V}^{\star}$ is the largest among all outputnulling subspaces of the triple $(A, B, C)$, so that

$$
\mathscr{V}^{\star} \supseteq \operatorname{ker}\left[\begin{array}{c}
C \\
C(A+B F) \\
\vdots \\
C(A+B F)^{n-1}
\end{array}\right] \quad \forall F \in \mathbb{R}^{n \times m} .
$$

We only need to show that there exists an $F \in \mathbb{R}^{n \times m}$ for which the inclusion (8) holds as an equality. This is the case for a friend of $\mathscr{V}^{\star}$. Indeed, if $F$ is a friend of $\mathscr{V}^{\star}$, there holds

$$
(A+B F) \mathscr{V}^{\star} \subseteq \mathscr{V}^{\star} \subseteq \operatorname{ker} C,
$$

so that $C \mathscr{V}^{\star}=\{0\}$, and $C(A+B F)^{k} \mathscr{V}^{\star} \subseteq C(A+$ $B F)^{k-1 \mathscr{V}^{\star}} \subseteq \ldots \subseteq C^{\mathscr{V}^{\star}}=\{0\}$, and we get

$$
\left[\begin{array}{c}
C \\
C(A+B F) \\
\vdots \\
C(A+B F)^{n-1}
\end{array}\right] \mathscr{V}^{\star}=\{0\} .
$$

Dualising this argument, one easily sees that the identity

$$
\mathscr{S}^{\star}=\min _{G \in \mathbb{R}^{p \times n}} \mathscr{R}(A+G C, B)
$$

also holds. We use the tools introduced in this section to prove the second main result of this contribution.

Theorem 3.1: Let the cheap LQ problem admit nonimpulsive solutions for all $x_{0} \in \mathbb{R}^{n}$. Then

$$
\mathscr{S}^{\star}=\mathscr{R}^{\star} \text {. }
$$

Proof: Suppose that the cheap LQ problem admits nonimpulsive solutions for all $x_{0} \in \mathbb{R}^{n}$. We must show that (9) holds. As already recalled, $\mathscr{R}^{\star}$ coincides with the intersection 
of $\mathscr{V}^{\star}$ and $\mathscr{S}^{\star}$. Thus, (9) is equivalent to saying that $\mathscr{S}^{\star} \subseteq$ $\mathscr{V}^{\star}$. Thus, we need to show that the inclusion

$$
\mathscr{R}(A+G C, B) \subseteq \mathscr{N}(A+B F, C)
$$

holds. This can be proved by showing that

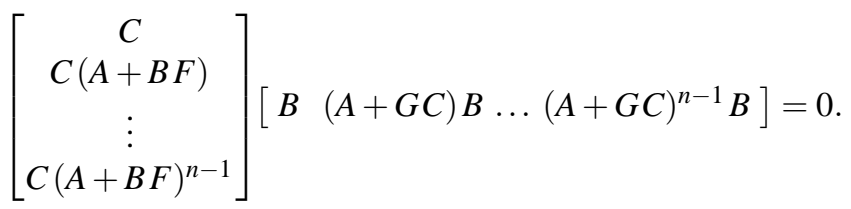

Every term of the matrix product can be written as

$$
C(A+B F)^{j}(A+G C)^{i} B
$$

for some $i, j \in\{0, \ldots, n-1\}$. We show that if $C e^{A t} B$ is identically zero, as established in the proof of Theorem 3.1, then each of these terms is zero. First, observe that $C e^{A t} B$ implies $C A^{l} B=0$ for all $l \in \mathbb{N}$. Then, $(A+G C)^{i}$ can be written as

$$
(A+G C)^{i}=A^{i}+M_{i} G C+\sum_{h=1}^{i-1} N_{i, h} C A^{h}
$$

for suitable matrices $M_{i}$ and $N_{i, 1}, \ldots, N_{i, i-1}$. Indeed, by induction we find

$$
\begin{aligned}
(A+G C)(A+G C)^{i}= & (A+G C)\left(A^{i}+M_{i} G C+\sum_{h=1}^{i-1} N_{i, h} C A^{h}\right) \\
= & A^{i+1}+A M_{i} G C+\sum_{h=1}^{i-1} A N_{i, h} C A^{h} \\
& +G C A^{i}+G C M_{i} G C+\sum_{h=1}^{i-1} G C N_{i, h} C A^{h} .
\end{aligned}
$$

Defining $M_{i+1}=A M_{i}+G C M_{i}$ and

$$
N_{i+1, h}= \begin{cases}A N_{i, h}+G C N_{i, h} & h \in\{0, \ldots, i-1\} \\ G & h=i\end{cases}
$$

we have

$$
(A+G C)^{i+1}=A^{i+1}+M_{i+1} G C+\sum_{h=1}^{i} N_{i+1, h} C A^{h} .
$$

In a similar way, we can show that

$$
(A+B F)^{j}=A^{j}+B F S_{j}+\sum_{k=1}^{j-1} A^{k} B T_{j, k}
$$

for suitable matrices $S_{j}$ and $T_{j, 1}, \ldots, T_{j, j-1}$. Hence,

$$
\begin{aligned}
C(A+B F)^{j}(A+G C)^{i} B \\
=C\left(A^{j}+B F S_{j}+\sum_{k=1}^{j-1} A^{k} B T_{j, k}\right)\left(A^{i}+M_{i} G C+\sum_{h=1}^{i-1} N_{i, h} C A^{h}\right) B \\
=C\left(A^{j+i}+A^{j} M_{i} G C+\sum_{h=1}^{i-1} A^{j} N_{i, h} C A^{h}\right. \\
\quad+B F S_{j} A^{i}+B F S_{j} M_{i} G C+\sum_{h-1}^{i-1} B F S_{j} N_{i, h} C A^{h} \\
\quad+\sum_{k=1}^{j-1} A^{k} B T_{j, k} A^{i}+\sum_{k=1}^{j-1} A^{k} B T_{j, k} M_{i} G C \\
\left.\quad+\sum_{k=1}^{j-1} A^{k} B T_{j, k} \sum_{h=1}^{i-1} N_{i, h} C A^{h}\right) B=0,
\end{aligned}
$$

since $C A^{l} B=0$ for all $l \in \mathbb{N}$.

\section{CONCLUDING REMARKS}

In this paper, we have shown that a fundamental question that remained open in [9], [10] on the connection between the generalised Riccati equation and the singular LQ problem has an affirmative answer, at least in the cheap case. In a forthcoming paper, it is shown that this result holds under a more general framework.

\section{REFERENCES}

[1] B.D.O. Anderson and J.B. Moore. Optimal Control: Linear Quadratic Methods. Prentice Hall International, London, 1989.

[2] G. Basile and G. Marro. Controlled and Conditioned Invariants in Linear System Theory. Prentice Hall, Englewood Cliffs, New Jersey, 1992.

[3] T. Chen and B.A. Francis. Spectral and inner-outer factorisations of rational matrices. SIAM Journal of Matrix Analysis and Applications, 10(1):1-17, 1989.

[4] A. Ferrante, G. Marro, and L. Ntogramatzidis, "A parametrization of the solutions of the finite-horizon LQ problem with general cost and boundary conditions". Automatica, 41(8): 1359-1366, 2005.

[5] A. Ferrante, and L. Ntogramatzidis, "A Unified Approach to the FiniteHorizon Linear Quadratic Optimal Control Problem". European Journal of Control, 13/5: 473-488, 2007.

[6] L. Ntogramatzidis, and A. Ferrante, "On the solution of the Riccati differential equation arising from the LQ optimal control problem". Systems \& Control Letters, 59:114-121, 2010.

[7] A. Ferrante, L. Ntogramatzidis, The generalised discrete algebraic Riccati equation in LQ optimal control. Automatica, 49(2):471-478, 2013.

[8] A. Ferrante, L. Ntogramatzidis, The extended symplectic pencil and the finite-horizon LQ problem with two-sided boundary conditions. IEEE Transactions on Automatic Control, 58(8), 2102-2107, 2013.

[9] A. Ferrante, and L. Ntogramatzidis, "The role of the generalised continuous algebraic Riccati equation in impulse-free continuous-time singular LQ optimal control". In Proceedings of the $52^{\text {nd }}$ Conference on Decision and Control (CDC 13), Florence, Italy, Dec 10-13, 2013.

[10] A. Ferrante, and L. Ntogramatzidis, "The generalized continuous algebraic Riccati equation and impulse-free continuous-time LQ optimal control". Automatica, 50(4):1176-1180, 2014.

[11] G. Freiling, G. Jank, and A. Sarychev. Non-blow-up conditions for Riccati-type matrix differential and difference equations. Results of Mathematics, 37:84-103, 2000.

[12] M.L.J. Hautus and L.M. Silverman. System structure and singular control. Linear Algebra and Its Applications, 50:369-402, 1983.

[13] V. Ionescu and C. Oară. Generalized continuous-time Riccati theory. Linear Algebra and Its Applications, 232:111-130, 1996.

[14] F.L. Lewis and V. Syrmos. Optimal Control. John Wiley \& Sons, New York, 1995.

[15] L. Ntogramatzidis, and G. Marro, "A parametrization of the solutions of the Hamiltonian system for stabilizable pairs". International Journal of Control, 78(7): 530-533, May 2005.

[16] D. Prattichizzo, L. Ntogramatzidis, and G. Marro, "A new approach to the cheap LQ regulator exploiting the geometric properties of the Hamiltonian system". Automatica, 44: 2834-2839, 2008.

[17] D. Rappaport and L.M. Silverman. Structure and stability of discretetime optimal systems. IEEE Transactions on Automatic Control, AC$16: 227-233,1971$.

[18] A. Saberi and P. Sannuti. Cheap and singular controls for linear quadratic regulators. IEEE Transactions on Automatic Control, AC32(3):208-219, March 1987.

[19] A. Saberi, P. Sannuti, and B.M. Chen. $H_{2}$ Optimal Control. System and Control Engineering. Prentice Hall International, London, 1995.

[20] A.A. Stoorvogel. The singular $\mathrm{H}_{2}$ control problem. Automatica, 28(3):627-631, 1992.

[21] A.A. Stoorvogel and A. Saberi. The discrete-time algebraic Riccati equation and linear matrix inequality. Linear Algebra Appl., 274:317$365,1998$.

[22] H.L. Trentelman, A.A. Stoorvogel, and M. Hautus. Control theory for linear systems. Springer, 2001. 
[23] M. Weiss, "Spectral and inner-outer factorisations through the constrained Riccati equation". IEEE Transactions on Automatic Control, AC-39(3):677-681, 1994.

[24] J.C. Willems, A. Kìtapçi, and L.M. Silverman. "Singular optimal control: a geometric approach". SIAM Journal of Control and Optimization, 24(2):323-337, March 1986.

[25] W.M. Wonham. Linear Multivariable Control: A Geometric Approach. Springer-Verlag, New York, 3 edition, 1985. 